Alexander A. Westphalen · Anna M. Russell

Mauro Buser · Claudine Rey Berthod · Pierre Hutter

Martina Plasilova · Hansjakob Mueller · Karl Heinimann

\title{
Evidence for genetic anticipation in hereditary non-polyposis colorectal cancer
}

Received: 26 October 2004/ Accepted: 14 January 2005 / Published online: 17 March 2005

(C) Springer-Verlag 2005

\begin{abstract}
Hereditary non-polyposis colorectal cancer (HNPCC) is an autosomal, dominantly inherited, colorectal cancer (CRC) predisposition syndrome caused by germline mutations in DNA mismatch repair (MMR) genes, predominantly $M L H 1$ and $M S H 2$. Thus far, only limited data exist on the occurrence of genetic anticipation in HNPCC, i.e. the earlier age at diagnosis of $\mathrm{CRC}$ in successive generations. Performing nonparametric distribution-free statistical analyses, we investigated 55 parent-child pairs who had been diagnosed with CRC and who came from 21 Swiss HNPCC families with characterised MMR germline mutation (15 in $M L H 1$ and 6 in MSH2). The overall median age at diagnosis was 43 years, with an interquartile range (IQR) of 14 and incidence ages ranging from 18 to 62 years. Descendants of HNPCC patients (median age at diagnosis 39 years, IQR =12) were found to be diagnosed with CRC significantly earlier than their parents $(47$ years, IQR $=10)$, with the median of the paired age difference amounting to 8 years $(\mathrm{IQR}=15$; $P<0.0001)$. Birth cohort effects could be excluded, since the same, statistically significant, age difference was also observed in the oldest offspring birth cohort (birth year $<1916 ; P=0.01)$. Genetic anticipation appeared to be more pronounced when the disease allele was transmitted through the father than through the mother (median age difference 11 vs. 4 years, respectively; both $P<0.01$ ). If confirmed in larger, ideally prospective studies, these results may have important implications for genetic
\end{abstract}

\footnotetext{
A. A. Westphalen · A. M. Russell · M. Buser · M. Plasilova · H. Mueller $\cdot$ K. Heinimann $(\bowtie)$

Research Group Human Genetics, Division of Medical Genetics, Centre for Biomedicine, University of Basel, Mattenstrasse 28, 4058 Basel, Switzerland

E-mail: karl.heinimann@unibas.ch

Tel.: + 41-61-2670777

Fax: +41-61-2670778

C. R. Berthod · P. Hutter

Unit of Genetics, Institut Central des Hopitaux Valaisans, Sion, Switzerland
}

counselling and clinical management of HNPCC families.

\section{Introduction}

Hereditary non-polyposis colorectal cancer (HNPCC) is an autosomal, dominantly inherited, colorectal cancer (CRC) predisposition syndrome, exhibiting high penetrance $(80 \%-85 \%)$ and accounting for $2 \%-10 \%$ of the total CRC burden (Lynch and de la Chapelle 1999). HNPCC patients typically present with, mostly rightsided, CRC at an early age (mean age 45 years) and often develop synchronous or metachronous CRC. In addition to $\mathrm{CRC}$, the tumour spectrum includes cancers of the endometrium, stomach, small bowel, ovary, ureter/renal pelvis, brain, hepatobiliary tract and skin. HNPCC is caused by mutations in DNA mismatch repair (MMR) genes (MSH2, MLH1, MSH6, PMS2), with $M L H 1$ and $M S H 2$ accounting for more than $90 \%$ of all germline mutations identified. Following inactivation of the wild-type allele in the tumour, MMR deficiency ensues, leading to genetic instability as exemplified by the occurrence of microsatellite instability caused by replication errors at short repetitive DNA sequences. The disease shows considerable interfamilial and intrafamilial phenotypic heterogeneity among $M L H 1 / M S H 2$ mutation carriers. Despite harbouring identical MMR gene mutations, disease severity and age at diagnosis often vary significantly between family members. In this context, genetic anticipation, a "phenomenon in which the age of onset of a disorder is reduced and/or the severity of the phenotype is increased in successive generations" (Strachan and Read 1999), has been postulated to occur in HNPCC. Thus far, only limited and controversial data are available on this issue, ranging from single case reports to a few systematic investigations in HNPCC families (Menko et al. 1993; 
Rodriguez-Bigas et al. 1996; Tsai et al. 1997; Vasen et al. 1994). In this study, we have examined 21 Swiss HNPCC kindreds with identified $M L H 1$ or $M S H 2$ germline mutations for the occurrence of genetic anticipation with regard to the age at diagnosis of CRC in parents and their descendants (first-degree kinship).

\section{Subjects and methods}

Out of 46 Swiss HNPCC families with an identified MMR gene mutation in $M L H 1$ or $M S H 2$, registered between 1990 and 2001 either at the Research Group Human Genetics, Division of Medical Genetics, Department of Clinical and Biological Sciences, Basel, or at the Unit of Genetics, Institut Central des Hôpitaux Valaisans, Sion, Switzerland, $26(57 \%)$ unrelated families were considered suitable for the study. Complete phenotypic information was available from medical and histopathological records regarding gender, year of birth, age at diagnosis, tumour type and the occurrence of additional (extracolonic) tumours.

The 26 families harboured either a pathogenic MLH1 $(n=18)$ or an MSH2 $(n=8)$ germline mutation. Out of the 126 individual patients, 91 parent-child pairs (PCPs) were created, each consisting of an affected parent and an affected child. Twenty-two parents had more than one child (range: 2-5 children) and 14 descendants also had affected children included in this study. In $145(79.7 \%) M L H 1 / M S H 2$ known or obligatory mutation carriers, the first tumour diagnosed was colorectal, compared with $37(20.3 \%)$ patients with an extracolonic tumour. For the study, only PCPs with children and parents affected with CRC (21 families; $n=55)$ were analysed. Based on the available clinical records, there was no indication that some of the patients participated in regular endoscopic surveillance programmes prior to the cancer diagnosis. The PCPs were treated as related data for the statistical analysis. Written informed consent was obtained from all individuals alive.

\section{Statistical analysis}

Nonparametric distribution-free tools were applied for statistical analysis. Related data were compared by applying the Wilcoxon matched-pairs signed-ranks test (referred to as paired Wilcoxon test). Independent data were compared with the Mann-Whitney U test. The disproportions of gender frequencies were analysed with the Binomial test. The median and the interquartile range (IQR) were used instead of the mean and the standard deviation, respectively. The significance level was set to $\leq 0.05(5 \%)$. All statistical tests were applied
Fig. 1 a Overall median age at diagnosis, grouped in 5-year age classes, comparing parents (black bars) with their descendants (grey bars) from 21 HNPCC kindreds with identified $\mathrm{MLH} 1$ or $\mathrm{MSH} 2$ germline mutations. b Box plot (including median and 10th, 25th, 75th and 90th percentile) of the paired age difference (years) between parents and descendants, grouped according to parental age at diagnosis of colorectal cancer $(<40$ below age $40,40-50$ between age 40 and $50,>50$ above age 50)
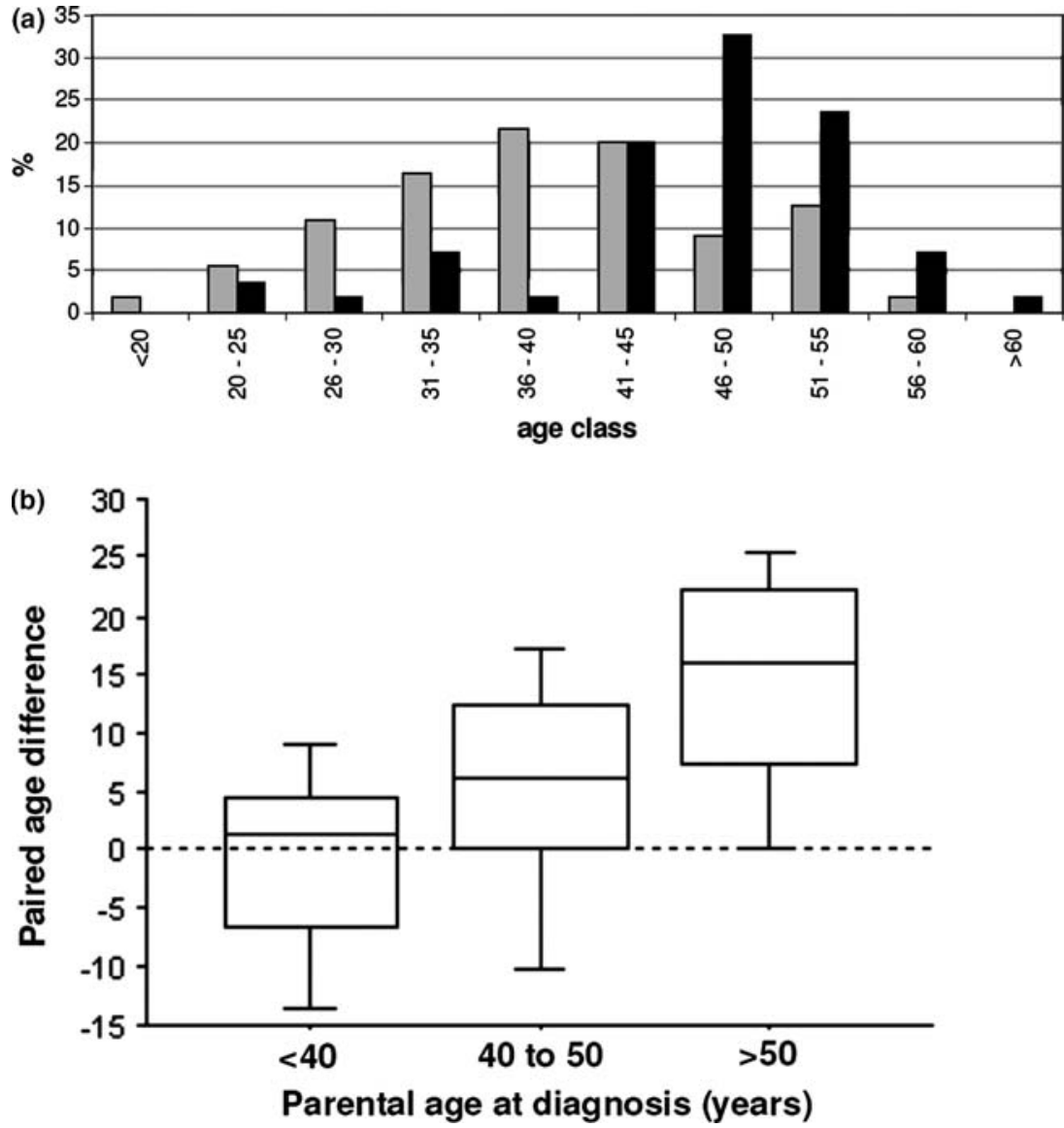
Table 1 Median age (years) at diagnosis of colorectal cancer (CRC) in parents and descendants, and according to MMR germline mutation. $P$-values are given for the respective difference in age at diagnosis between parents and descendants (IQR interquartile range and $\mathrm{n}$ the number of PCPs)

\begin{tabular}{|c|c|c|c|c|c|c|c|c|c|}
\hline Subjects & \multicolumn{3}{|c|}{ Overall } & \multicolumn{3}{|c|}{$M L H 1(n=41)$} & \multicolumn{3}{|c|}{$M S H 2(n=14)$} \\
\hline Descendants (D) & 39 & 12 & $18-58$ & 38 & 10 & $18-55$ & 44 & 16 & $29-58$ \\
\hline Difference P-D & 8 & 15 & -14 to 30 & 11 & 15 & -14 to 30 & 2 & 8 & -12 to 20 \\
\hline$n$ & & 55 & & & & & & 14 & \\
\hline$P$ & & $<0.0001$ & & & $<0.001$ & & & 0.12 & \\
\hline
\end{tabular}

in their two-sided form. The software-packages used for the statistical analysis were "RS/1" (Research System 1), Version 6.1 (Brooks Automation), and "StatXact", Version 4 (Cytel Software Corporation).

\section{Results}

To assess the occurrence of genetic anticipation in HNPCC, 55 PCPs affected with CRC and coming from 21 Swiss HNPCC kindreds with identified MLH1 $(n=15)$ or $\mathrm{MSH} 2 \quad(n=6)$ germline mutations were examined. The PCPs encompassed $52(62.7 \%)$ male and $31(37.3 \%)$ female known or obligatory mutation carriers. Following CRC diagnosis, $24(28.9 \%)$ patients also developed metachronous cancers (range 1-4 tumours) encompassing those of the colorectum $(n=15$; $62.5 \%)$, small intestine $(n=2 ; 8.3 \%)$, stomach $(n=1$; $4.2 \%)$ and other sites $(n=6 ; 25 \%)$. Median age at diagnosis did not significantly differ between genders, either in parents $(P=0.42)$ or in descendants $(P=0.23)$.

The overall median age at diagnosis was 43 years $(\mathrm{IQR}=14)$, with incidence ages ranging from 18 to 62 years (Fig. 1a). Taking the median of the paired age differences, descendants (39 years, IQR $=12$ ) were diagnosed 8 years earlier than their parents (47 years, $\mathrm{IQR}=10.0$ ), the difference being statistically significant (paired Wilcoxon test, $P<0.0001$ ), with $76 \%, 9 \%$ and $15 \%$ of descendants being diagnosed at an earlier, same, and later age, respectively (Table 1). Moreover, the paired age difference was more marked if the parents had developed CRC at a later age (Fig. 1b).

A comparison of the median age at diagnosis according to the gene mutated revealed that descendants with an $M L H 1$ germ-line mutation were diagnosed statistically significantly earlier than those with an $\mathrm{MSH} 2$ mutation (38 vs. 43.5 years, $P<0.05$ ) in contrast to parents ( 47 vs. 48 years, $P=0.91$ ). Similarly, PCPs with a MLH1 mutation displayed a significant median of the paired age difference $(P<0.01)$. The median of the paired age difference in $M S H 2$ PCPs with CRC did not reach statistical significance, possibly because of the small sample size $(n=14 ; P=0.12$; Table 1$)$.

When subdividing PCPs according to parental transmission, sons and daughters who inherited the disease allele from their father showed a significantly increased median age difference of 11 years $(P<0.001)$ compared with 4 years in children who had inherited the germline mutation from the mother $(P<0.01$; Table 2$)$. Importantly, median age at diagnosis did not significantly differ between mothers ( 45 years, IQR $=14$ ) and fathers (47 years, IQR $=10 ; P=0.5$ ).

In order to test whether the observation of apparent anticipation could reflect a birth cohort bias of ascertainment, we grouped the PCPs according to the birth year of the children and created three birth cohorts: (1) those born before $1916(n=9)$, (2) those born between 1916 and $1936(n=20)$ and (3) those born after 1936 $(n=24)$. As depicted in Table 3, the median of the paired age difference remained statistically significant in the first (15 years; $P=0.01$ ) and third cohort ( 9 years; $P=0.001)$. In the second cohort, the median age difference amounted to 5 years but just missed statistical significance (two-sided $P=0.06$ ).

\section{Discussion}

In this study, we provide significant evidence for genetic anticipation in 55 PCPs coming from 21 Swiss HNPCC kindreds with characterised $M L H 1$ or $M S H 2$ germline mutations. Compared with the parents, descendants developed CRC 8 years earlier, in particular if the parents had developed cancer at a later age.

Other than the initial observation by Warthin (1925), only four studies deal in detail with the occurrence of genetic anticipation in HNPCC, i.e. the progressive decrease in age of onset of CRC in successive generations. Although three investigations have ob-

Table 2 Median of the paired age difference according to the sex of the transmitting parent

\begin{tabular}{lllll}
\hline Transmission & $n$ & $\begin{array}{l}\text { Age difference } \\
\text { (years) }\end{array}$ & IQR & Range \\
\hline Mother to child $(P<0.01)$ & 19 & 4 & 13.75 & -13 to 25 \\
Mother to daughter & 7 & 3 & 11.75 & -13 to 20 \\
Mother to son & 12 & 4.5 & 14.5 & -10 to 25 \\
Father to child $(P<0.001)$ & 34 & 11 & 17 & -14 to 30 \\
Father to daughter & 13 & 11 & 14 & -14 to 17 \\
Father to son & 21 & 7 & 18.5 & -7 to 30 \\
\hline
\end{tabular}


Table 3 Median age (years) at diagnosis of CRC in parents and descendants according to the birth year of the children

\begin{tabular}{|c|c|c|c|c|c|c|c|c|c|}
\hline \multirow[t]{2}{*}{ Subjects } & \multicolumn{3}{|c|}{$\begin{array}{l}\text { Birth cohort 1: born before } \\
1916\end{array}$} & \multicolumn{3}{|c|}{$\begin{array}{l}\text { Birth cohort 2: born between } \\
1916 \text { and } 1936\end{array}$} & \multicolumn{3}{|c|}{ Birth cohort 3: born after 1936} \\
\hline & Age & IQR & Range & Age & IQR & Range & Age & IQR & Range \\
\hline Parents $(\mathrm{P})$ & 55 & 14 & 34 to 62 & 46 & 7 & 41 to 55 & 49 & 15 & 22 to 60 \\
\hline Descendants (D) & 40 & 8 & 32 to 47 & 43 & 19 & 22 to 58 & 36 & 10 & 18 to 54 \\
\hline Difference P-D & 15 & 16 & -1 to 20 & 5 & 13 & -14 to 25 & 9 & 15 & -14 to 30 \\
\hline$P$ & & 0.01 & & & 0.06 & & & 0.001 & \\
\hline
\end{tabular}

served anticipation in HNPCC kindreds fulfilling the Amsterdam Criteria I (ACI), they cannot be directly compared with our study, since (1) no statistical tests were applied, or (2) investigations were carried out either before the actual discovery of the genes responsible (Menko et al. 1993; Vasen et al. 1994) or afterwards, but without molecular genetic characterisation (Rodriguez-Bigas et al. 1996). The fourth study by Tsai et al. (1997) analysed a total of 67 ACI-positive PCPs (38 families) with $M S H 2$ and $M L H 1$ germline mutations characterised in 14 pairs ( 7 families) of them. However, they could not detect any difference in mean age at diagnosis between generations in any of the subgroups analysed. This apparent contradiction to our findings could be attributable to the small sample size (14, compared with 55 PCPs in our study) leading to a type II error (failure to reject the null hypothesis). In addition, the comparison might be hampered by differences in the way in which data were collected and the time window analysed.

In contrast to the findings by Tsai et al. (1997), a possible birth cohort bias of ascertainment could not be confirmed in our study group (Table 3): the median of the paired age difference remained statistically significant in the youngest and oldest birth cohort ( 9 years, $P<0.001$, and 15 years, $P<0.01$, respectively), and just failed to reach significance in the middle birth cohort (5 years, $P=0.06$ ). On the other hand, PCPs with cancers other than CRC $(n=36)$, which were excluded from the original study, revealed a clear birth cohort effect (data not shown).

Intriguingly, genetic anticipation in our study population was more pronounced if the disease allele was transmitted through the father than through the mother ( 9.5 vs. 4 years paired median age difference, respectively; Table 2). This finding does not appear to be attributable to differences in median age at diagnosis between fathers and mothers, as this was similar in both parental groups (47 vs. 45 years; $P=0.5$ ). Green et al. (2002) have observed similar parent-of-origin effects in a large HNPCC kindred with a founder mutation in MSH2: females who inherited the mutation from their father had an increased risk of developing CRC and of dying of cancer compared with females carrying a maternally transmitted mutation. It remains to be seen, however, whether this apparent parental transmission effect in our study group is merely the result of ascertainment bias or may actually reflect a true biological phenomenon. If so, it is tempting to speculate that this effect is related to differences in male and female gametogenesis, in particular given the greater number of cell divisions during spermatogenesis and continuous replication throughout adulthood. Given the data implicating $M L H 1$ in the meiotic recombination process (Eaker et al. 2002; Wang et al. 1999) and in analogy to genetic anticipation observed in trinucleotide repeat disorders such as Huntington's disease (Quinn and Schrag 1998), MMR (haplo) insufficiency in the germline could be hypothesised to lead to anticipation via low level repeat instability.

Despite the evidence for genetic anticipation presented here, there are several caveats to this retrospective study. First of all, we cannot exclude an ascertainment bias, since our study population represents a highly selected group of patients. In particular, selection bias might have resulted from either under-representation of "younger parent-older child" pairs in which the parent had died before producing a "complete" family or from selection for descendants with early onset CRC. Furthermore, the significant difference in age at diagnosis of CRC observed between the generations could (1) reflect changes in environmental factors such as dietary and life style habits, (2) be attributable, at least in part, to earlier and better diagnosis progressively over time and/or (3) greater awareness/anxiety in descendants. It is therefore mandatory that these findings are confirmed in larger, ideally prospective studies of HNPCC kindreds. These could also allow an assessment of the occurrence of genetic anticipation for other HNPCC related tumours, particularly endometrial cancer.

In summary, we have presented significant evidence that genetic anticipation occurs in HNPCC families harbouring MMR gene mutations, with descendants being diagnosed with CRC 8 years earlier than their parents. Intriguingly, this effect seems to be more pronounced if the disease allele is transmitted through the father. If our findings concerning genetic anticipation in general and paternal transmission in particular are confirmed, they may have important implications for the genetic counselling and clinical management of MMR gene mutation carriers and their offspring.

Acknowledgments We thank all the patients and families, who participated in this study, and their respective doctors for contributing clinical information. We are grateful to Michele Attenhofer and Marianne Haeusler for excellent technical assistance and 
to Dr. Ian Tomlinson for critical reading of the manuscript. This work was supported by grants from the Swiss National Science Foundation (no. 3200-067571) and Swiss Cancer League/Oncosuisse (no. 01358-03-2003).

\section{References}

Eaker S, Cobb J, Pyle A, Handel MA (2002) Meiotic prophase abnormalities and metaphase cell death in MLH1-deficient mouse spermatocytes: insights into regulation of spermatogenic progress. Dev Biol 249:85-95

Green J, O'Driscoll M, Barnes A, Maher ER, Bridge P, Shields K, Parfrey PS (2002) Impact of gender and parent of origin on the phenotypic expression of hereditary nonpolyposis colorectal cancer in a large Newfoundland kindred with a common MSH2 mutation. Dis Colon Rectum 45:1223-1232

Lynch HT, Chapelle A de la (1999) Genetic susceptibility to nonpolyposis colorectal cancer. J Med Genet 36:801-818

Menko FH, Te Meerman GJ, Sampson JR (1993) Variable age of onset in hereditary nonpolyposis colorectal cancer: clinical implications. Gastroenterology 104:946-947
Quinn N, Schrag A (1998) Huntington's disease and other choreas. J Neurol 245:709-716

Rodriguez-Bigas MA, Lee PH, Malley L, Weber TK, Suh O, Anderson GR, Petrelli NJ (1996) Establishment of a hereditary nonpolyposis colorectal cancer registry. Dis Colon Rectum 39:649-653

Strachan T, Read AP (1999) Human molecular genetics 2. Wiley, New York

Tsai YY, Petersen GM, Booker SV, Bacon JA, Hamilton SR, Giardiello FM (1997) Evidence against genetic anticipation in familial colorectal cancer. Genet Epidemiol 14:435-446

Vasen HF, Taal BG, Griffioen G, Nagengast FM, Cats A, Menko FH, Oskam W, Kleibeuker JH, Offerhaus GJ, Khan PM (1994) Clinical heterogeneity of familial colorectal cancer and its influence on screening protocols. Gut 35:1262-1266

Wang TF, Kleckner N, Hunter N (1999) Functional specificity of MutL homologs in yeast: evidence for three Mlh1-based heterocomplexes with distinct roles during meiosis in recombination and mismatch correction. Proc Natl Acad Sci USA 96:13914 13919

Warthin AS (1925) The further study of a cancer family. J Cancer Res 9:279-286 